\title{
RNA device rewires cellular networks
}

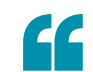

RNA is an apt

molecule for

such devices,

given its

versatile sensing

functions and

the ease with

which RNA

molecules can

be designed.
Synthetic devices that translate molecular inputs into gene expression changes are valuable for manipulating cellular behaviour, but until now these circuits have only been able to respond to a limited set of inputs, such as transcription factors. However, a new synthetic device based on RNA can respond sensitively to alterations in the levels of endogenous signalling molecules, and so can potentially link disease-associated pathways to cell fate decisions.

At the heart of the new device is an RNA aptamer, an RNA molecule that alters its conformation and function upon binding to a particular protein ligand. In the new study, an RNA aptamer is inserted into the intron of a three-exon minigene. On binding of the aptamer to a cellular factor, the RNA alters its conformation, and in so doing affects the alternative

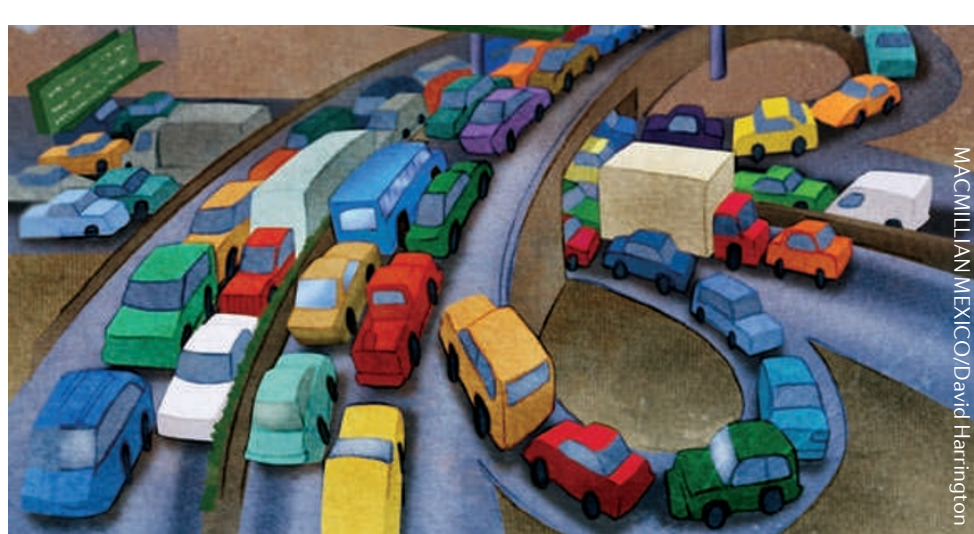

splicing of the middle exon of the minigene. This splicing event has crucial consequences: the middle exon contains a stop codon, and so its removal leads to the upregulation of an output gene that is under the control of the minigene product. This sensor-actuator construct therefore has the potential to link changes in intracellular protein concentration (via the sensor module, represented by the aptamer) to gene expression changes (via the actuator minigene and the output gene).

The flexibility of the synthetic device was shown by applying it in different settings in human cell lines. In the first application, cells were stably transfected with devices in which two aptamers responded, respectively, to changes in the levels of two subunits of nuclear factor- $\kappa \mathrm{B}(\mathrm{NF}-\kappa \mathrm{B})$. In the second example, the aptamer was

As the examples above show, these As the examples above show, these particular devices can also easily be adapted for combinatorial use in clinical applications (such as gene or cellular therapy, or in diagnostics) or as drug discovery tools.

Tanita Casci

Senior Editor, Nature Reviews Genetics

ORIGINAL RESEARCH PAPER Culler, S. J., Hoff, K. G. \& Smolke, C. D. Reprogramming cellular behavior with RNA controllers responsive to endogenous proteins. Science 330, 1251-1255 (2010) 